\title{
Neutrino-nucleus cross section in the impulse approximation regime
}

\author{
Omar Benhar $^{\mathrm{ab}}$, Nicola Farina ${ }^{\mathrm{b}}$

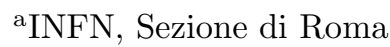 \\ Piazzale Aldo Moro, 2. I-00185 Roma, Italy \\ bDipartimento di Fisica, Università "La Sapienza" \\ Piazzale Aldo Moro, 2. I-00185 Roma, Italy
}

In the impulse approximation regime the nuclear response to a weakly interacting probe can be written in terms of the measured nucleon structure fuctions and the target spectral function, yielding the energy and momentum distribution of the constituent nucleons. We discuss a calculation of charged current neutrino-oxygen interactions in the quasielastic channel, carried out within nuclear many body theory. The proposed approach, extensively and successfully employed in the analysys of electron-nucleus scattering data, allows for a parameter free prediction of the neutrino-nucleus cross section, whose quantitative understanding will be critical to the analysis of the next genaration of high precision neutrino oscillation experiments.

\section{Introduction}

In experimental searches of oscillations, neutrinos are detected trough their interactions with nucleons bound in nuclei (e.g. oxygen). Therefore, the knowledge of the neutrino-nucleus cross section at a fully quantitative level will be critical to reduce the systematic uncertainty of the next generation of high precision measurements.

Accurate calculations of the weak nuclear response at moderate momentum transfer can be carried out within nuclear many body theory (NMBT) using nonrelativistic wave functions to describe the target initial and final states and expanding the current operator in powers of $(|\mathbf{q}| / m)$, $\mathbf{q}$ and $m$ being the momentum transfer and the nucleon mass, respectively. Applications of this approach to the analysis of electron scattering data has been very successful (for a review see, e.g., ref. [1]).

At the higher values of $|\mathbf{q}|$ corresponding to energies of the beam particles in the few GeV region, the description of the final states in terms of nonrelativistic nucleons is no longer possible, and some simplifying assumptions have to be made in order to take into account both the presence of relativistic particles and the occurrence of inelastic processes.
An approximation scheme widely employed to describe the region of high momentum transfer is based on the idea that, as the beam particle probes a region of extension $\sim 1 /|\mathbf{q}|$ of the target, at large $|\mathbf{q}|$ the scattering process involves only one nucleon, the remainig A-1 particles acting as spectators.

In the simplest implementation of this scheme, usually referred to as impulse approximation (IA), the hadronic state produced at the weak interaction vertex is assumed to be totally decoupled from the recoiling system, so that the description of its motion reduces to a purely kinematical problem. On the other hand, the target intial state, as well as the final state of the spectator particles, can be safely treated within NMBT using nonrelativistic dynamics.

In Section 2, after giving the expression of the neutrino-nucleus cross section in the IA regime, we briefly outline a theoretical approcah, based on NMBT, that allows for both a realistic description of the initial state and the inclusion of corrections arising from final state interactions (FSI). The results of calculation for the test case of quasielastic charged current interactions with oxygen are presented in Section 3, while Section 4 is devoted to a summary and the conclusions. 


\section{The neutrino-nucleus cross section in the impulse approximation regime}

The Born approximation cross section of the weak charged current process

$\nu_{\ell}+A \rightarrow \ell^{-}+X$,

where $A$ and $X$ denote the target nucleus and the undetected hadronic final state, respectively, can be written in the form

$\frac{d \sigma}{d \Omega_{\ell} d E_{\ell}}=\frac{G^{2}}{32 \pi^{2}} \frac{\left|\mathbf{k}^{\prime}\right|}{|\mathbf{k}|} L_{\mu \nu} W^{\mu \nu}$.

In the above equation, $G$ is the Fermi coupling constant, $E_{\ell}$ is the energy of the final state lepton and $\mathbf{k}$ and $\mathbf{k}^{\prime}$ are the neutrino and charged lepton momentum, respectively. The tensor $L_{\mu \nu}$ is fully specified by the kinematical variables of the leptons, while $W^{\mu \nu}$ is defined in terms of the target initial and final states and current according to

$$
\begin{aligned}
W^{\mu \nu}=\sum_{X}\left\langle A\left|J^{\mu^{\dagger}}\right| X\right\rangle\left\langle X\left|J^{\nu}\right| A\right\rangle & \\
& \times \delta^{(4)}\left(P_{A}+q-P_{X}\right),
\end{aligned}
$$

$P_{A}, P_{X}$ and $q$ being the target initial and final four momenta and the four momentum transfer, respectively.

The IA scheme outlined in Section 1 is based on the assumptions that: i) the target nucleus is seen by the neutrino as a collection of individual nucleons and ii) the hadron produced at the weak interaction vertex is unaffected by either Pauli blocking or final state interactions (FSI) with the spectator nucleons.

According to this picture, the state $|X\rangle$ appearing in Eq.(3) can be written in a simple factorized form and the tensor $W^{\mu \nu}$ of Eq.(3) reduces to (see, e.g. ref. [3])

$W_{I A}^{\mu \nu}=\int d^{4} p P(p) w^{\mu \nu}(\widetilde{p}, \widetilde{q})$,

where $P(p)\left(p \equiv\left(p_{0}, \mathbf{p}\right)\right)$ is the nuclear spectral function [2], yielding the probability of removing a nucleon of momentum $\mathbf{p}$ from the target leaving the residual system with energy $E=m-p_{0}$. Eq. (41) shows that the IA formalism allows one to describe scattering off a bound nucleon carrying four momentum $p$ in terms of the tensor $w^{\mu \nu}$, describing the weak charged current interactions of a nucleon in free space. Binding effects are taken care of by replacing the bound nucleon four momentum $p \equiv\left(p_{0}, \mathbf{p}\right)$ with $\widetilde{p} \equiv\left(\sqrt{\mathbf{p}^{2}+m^{2}}, \mathbf{p}\right)$ and the four momentum tranfer $q \equiv(\nu, \mathbf{q})$ with $\widetilde{q}=p-\widetilde{p}+q .345$.

The IA cross section can be obtained from Eqs.(2) and (4) using the available parametrizations of the structure functions (both elastic and inelastic) entering the definition of $w^{\mu \nu}$ and the nuclear spectral functions resulting from calculations carried out within nuclear many body theory (NMBT) 6.

NMBT also provides a consistent framework to include corrections to the IA in a systematic fashion. The effects of FSI, that have long been recognized to be large in both inclusive and exclusive electron-nucleus scattering processes (a series of review papers on electron-nucleus scattering can be found in ref. [1]), can be taken into account within the approach of ref. [3, based on the assumptions that i) the hadron produced at the weak interaction vertex moves along a straight trajectory with constant velocity (eikonal approximation) and ii) the spectator nucleons are seen as a collection of fixed scattering centers (frozen spectators approximation). The resulting cross section can be written in terms of the IA results according to

$\frac{d \sigma_{A}}{d \Omega_{\ell} d E_{\ell}}=\int d E_{\ell}^{\prime} f_{q}\left(E_{\ell}-E_{\ell}^{\prime}\right)\left(\frac{d \sigma}{d \Omega_{\ell} d E_{\ell}^{\prime}}\right)_{I A}$

The above equation shows that the the occurrence of FSI leads to a redistribution of the strength dictated by the folding function $f_{q}(E)$, whose shape is strongly affected by nucleon-nucleon correlations [3]. In absence of FSI $f_{q}(E) \rightarrow \delta(E)$ and the IA cross section is recovered.

\section{Results}

We have computed the cross section of the quasielastic process

$\nu_{e}+{ }^{16} O \rightarrow e^{-}+p+X$,

where $X$ denotes the recoiling spectator system. The spectral function apperaing in Eq.(4) has 
been obtained within the Local Density Approximation (LDA) 6], while for the Dirac, Pauli and axial form factors entering the definition of the nucleon tensor $w^{\mu \nu}$ we have used the simple dipole parametrization.

The folding function has been evaluated using a parametrization of the measured nucleon-nucleon scattering amplitude [7] and Monte Carlo configurations sampled from the probability distribution associated with the realistic oxygen wave function of ref. 8 .

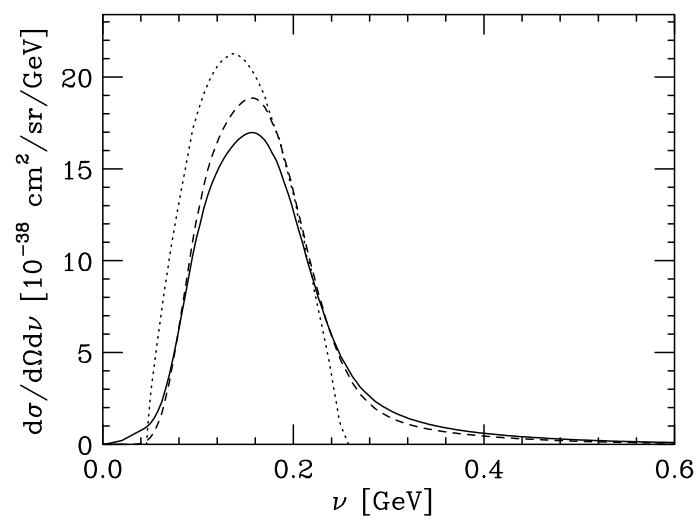

Figure 1. Differential cross section $d \sigma / d \Omega_{e} d E_{e}$ for neutrino energy $E=1 \mathrm{GeV}$ and electron scattering angle $\theta_{e}=30^{\circ}$. The IA results are represented by the dashed line, while the solid line correspond to the full calculation, including the effects of FSI. The dotted line shows the prediction of the FG model with Fermi momentum $k_{F}=221 \mathrm{MeV}$ and average separation energy $\epsilon=35 \mathrm{MeV}$.

Fig. 1shows the cross section corresponding to neutrino energy $E=1 \mathrm{GeV}$, and electron scattering angle $\theta_{e}=30^{\circ}$, plotted as a function of the energy transfer $E-E_{e}$. Comparison between the solid and dashed lines shows that the inclusion of FSI results in a sizable redistribution of the IA strength, leading to a quenching of the quasielastic peak and to the enhancement of the tails. For reference, we also show the cross section predicted by the Fermi gas (FG) model with Fermi momentum $k_{F}=221 \mathrm{MeV}$ and average separation energy $\epsilon=35 \mathrm{MeV}$. It appears that nuclear dynamics, neglected in the oversimplified picture in terms of noninteracting nucleons, plays a relevant role.

It has to be pointed out that the approach of ref. 3, while including dynamical correlations in the final state, does not take into account statistical correlations, leading to Pauli blocking of the phase-space available to the outgoing nucleon.

A rather crude prescription to include the effect of Pauli blocking amounts to modifying the spectral function according to

$P(p) \rightarrow P(p) \theta\left(|\mathbf{p}+\mathbf{q}|-k_{F}\right)$

where $k_{F}$ is the average nuclear Fermi momentum, defined as

$k_{F}=\int d^{3} r \rho_{A}(\mathbf{r}) k_{F}(\mathbf{r})$,

with $k_{F}(\mathbf{r})=\left(3 \pi^{2} \rho_{A}(\mathbf{r}) / 2\right)^{1 / 3}, \rho_{A}(\mathbf{r})$ being the measured nuclear density distribution. Eq.(3i) yields $k_{F}=209 \mathrm{MeV}$. Note that, unlike the spectral function, the quantity defined in Eq.(17) does not describe intrinsic properties of the target only, as it depends explicitely upon the momentum transfer.

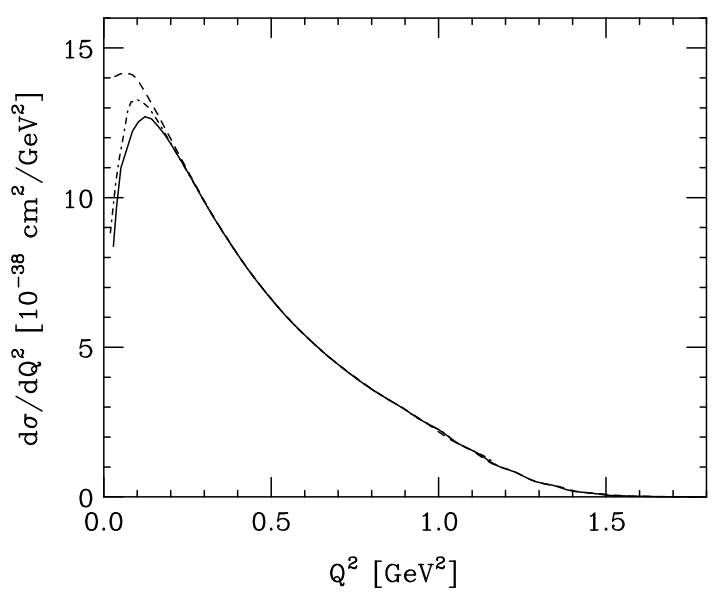

Figure 2. Differential cross section $d \sigma / d Q^{2}$ for neutrino energy $E=1 \mathrm{GeV}$. The dot-dash line shows the IA results, while the solid and dashed lines have been obtained using the modified spectral function ef Eq.(7), with and without inclusion of FSI, respectively. 
The effect of Pauli blocking is hardly visible in the differential cross sections shown in Fig. 1] as the kinematical setup corresponds to $Q^{2} \sim 2.4$ $\mathrm{GeV}^{2}$ at the quasielastic peak. On the other hand, it becomes very large at lower $Q^{2}$.

Fig. 2 shows the calculated differential cross section $d \sigma / d Q^{2}$ for neutrino energy $E=1 \mathrm{GeV}$. The dot-dash and dashed lines correspond to the IA results with and without inclusion of Pauli blocking, respectively. It clearly appears that the effect of Fermi statistic in suppressing scattering at $Q^{2}<.2 \mathrm{GeV}^{2}$ is very large and must be taken into account. The results of the full calculation, in which FSI are also included, are displayed as a full line. The results of Fig. 2 suggest that Pauli blocking and FSI may explain the deficit of the measured cross section at low $Q^{2}$ with respect to the predictions of Monte Carlo simulations [9].

The energy spectrum of the produced electrons obtained using the modified spectral function of Eq. (7) is displayed in Fig. 3. The solid and dashed lines corresponds to the results of calculations carried out with and without FSI. As in Fig. 11 it appears that inclusion of FSI results in a redistribution of the strength, leading to a quenching in the region of the peak and to the appearance of a tail at large energy, extending all the way to the boundary of the kinematically allowed region.

\section{Conclusions}

NMBT provides a parameter free and computationally viable framework to study the nuclear response to weakly interacting probes, whose quantitative understanding will be critical to the analysis of the next genaration of high precision neutrino oscillation experiments.

In the IA scheme, which is likely to be applicable to describe scattering of few $\mathrm{GeV}$ neutrinos, NMBT allows for a consistent treatment of dynamical nucleon-nucleon correlations, that largely affect both the nucleon spectral function and FSI. Electron scattering experiments have provided overwhelming evidence that the independent particle model fails to explain the data and inclusion of correlation effects, leading to a sizable redistribution of the strength, is indeed

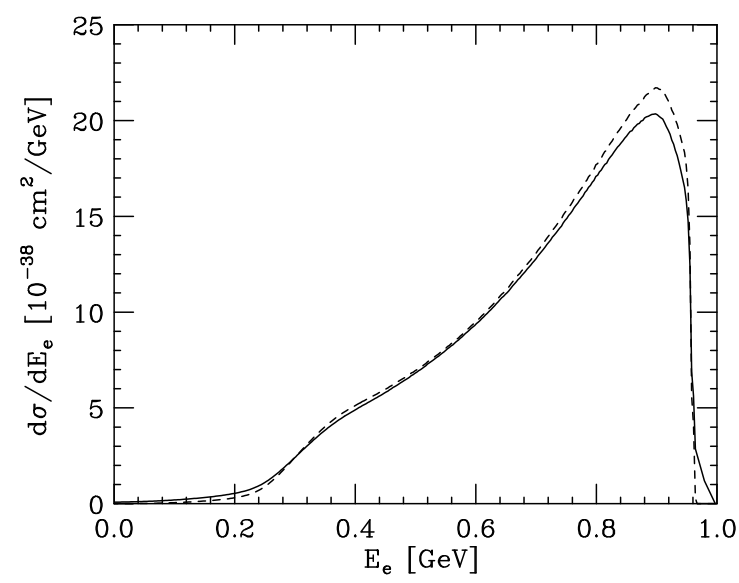

Figure 3. Energy spectrum of the produced electrons in charged current interactions at neutrino energy $E=1 \mathrm{GeV}$. The solid and dashed lines have been obtained including the effect of Pauli blocking with and without FSI, respectively.

required.

As a final remark, it has to be emphasized that the extension of the approach described in this paper to include exclusive as well as inelastic channels, along the lines of the work done for electron-nucleus scattering, is straightforward.

\section{REFERENCES}

1. Modern Topics in Electron Scattering, Editors B. Frois and I. Sick (World Scientific, Singapore, 1991).

2. O. Benhar, A. Fabrocini and S. Fantoni, Nucl. Phys. A505 (1989) 267.

3. O. Benhar et al, Phys. Rev. C 44 (1991) 2328.

4. T. de Forest, Jr., Nucl. Phys. A392 (1983) 232.

5. O. Benhar and V.R. Pandharipande, Phys. Rev. C 47 (1993) 2218.

6. O. Benhar, A. Fabrocini, S. Fantoni and I. Sick, Nucl. Phys. A579 (1994) 493.

7. T.G. O'Neill, private communication.

8. S.C. Pieper, R.B. Wiringa and V.R. Pandharipande, Phys. Rev. C 46 (1992) 1741.

9. T. Ishida, Nucl. Phys. B (Proc. Suppl.) 112 (2002) 132. 